\title{
Salames tipo Milano elaborados com fibras de subprodutos da produção de vinho tinto
}

\author{
Milano type salami elaborated with fibers of red wine byproducts
}

\author{
Andressa Cristina Gaione Mendes ${ }^{\mathrm{I}}$ Daynna Miranda Rettore ${ }^{\mathrm{II}}$ Alcinéia de Lemos Souza Ramos ${ }^{\mathrm{III}}$ \\ Simone de Fátima Viana da Cunha ${ }^{\mathrm{IV}}$ Luis Carlos de Oliveira ${ }^{\mathrm{III}}$ Eduardo Mendes Ramos ${ }^{\mathrm{II}}{ }^{*}$
}

RESUMO

Foram avaliados os efeitos da adição da farinha de subprodutos da produção de vinhos, em diferentes concentrações (1, 2 e 3\%), na produção de salame tipo Milano. Os processos de fermentação e secagem foram acompanhados por análises físico-químicas ( $\mathrm{pH}$ e atividade de água), químicas (acidez titulável, índice de peróxido, índice de TBARS e nitrito residual) e físicas (perda de peso) e os produtos acabados avaliados quanto à composição centesimal. Maiores adições de fibra reduziram $(P<0,05)$ os valores de $\mathrm{pH}$ e aumentaram o teor de ácido lático da massa cárnea durante a maturação, com consequente aumento nas perdas de peso e redução na atividade de água dos produtos. A presença de fibras não influenciou $(P>0,05)$ os níveis de nitrito residual e nem os valores de índice de peróxido, porém reduziu a evolução da oxidação lipídica, medida pelo índice de TBARS, do produto durante a maturação. Apesar de promover $(P<0,05)$ alterações no teor de cinzas, a presença de fibras não afetou $(P>0,05)$ os valores de umidade, proteína e lipídeos. A presença de fibras alimentares e compostos antioxidantes nas fibras da cultivar 'Syrah' permitiu a sua aplicação no processamento de salames com benefícios tecnológicos e nutricionais.

Palavras-chave: fibra alimentar, produto fermentado, oxidação lipídica.

\section{ABSTRACT}

The effects of the addition of wine byproducts flour in different concentrations (1, 2 and 3\%) on the production of salamitype Milano were studied. Fermentation and dry-curing processes were followed by physical (weight loss), physico-chemical ( $\mathrm{pH}$ and water activity) and chemical (acidity, peroxide values, TBARS index and residual nitrite level) analysis. The proximal composition of the finished products was also evaluated. Higher additions of fiber reduced $(P<0.05) \mathrm{pH}$ values and increased lactic acid content of the meat mixture during maturation, with consequent increase in weight loss and reduction in the water activity of the products. The presence of fibers did not affected $(P>0.05)$ the residual nitrite levels neither the peroxide values of the product during ripening, but reduced the development of lipid oxidation measured by TBARS values. The presence of fibers increased $(P<0.05)$ ash content, but did not affect $(P>0.05)$ values of moisture, protein and lipids. The presence of dietary fiber and antioxidant compounds in the Syrah fibers allowed the application in processing salamis with technological and nutritional benefits.

Key words: dietary fiber, fermented product, lipid oxidation.

\section{INTRODUÇÃO}

Atualmente, a fibra alimentar ou dietética é um ingrediente largamente utilizado no desenvolvimento de produtos com apelo nutricional, devido a sua significância na promoção da saúde e impacto tecnológico no produto. A Associação Dietética Americana recomenda uma ingestão de 25 a $30 \mathrm{~g}$ de fibra alimentar por dia para adultos (VERMA \& BANERJEE, 2010), tendo a sua importância devido a estas atuarem na prevenção de diversas doenças, como diabetes, cólon irritável, câncer de cólon, diverticulite, doenças cardiovasculares e gastrointestinais, entre outras (RODRÍGUEZ et al., 2006; VERMA \& BANERJEE, 2010).

A maioria dos alimentos cárneos processados não tem quantidades mínimas de fibra alimentar. Salames, por exemplo, além de não conter fibras podem conter até $30 \%$ de lipídeos no

IPrograma de Pós-graduação em Ciência dos Alimentos, Departamento de Ciência dos Alimentos (DCA), Universidade Federal de Lavras (UFLA), Lavras, MG, Brasil.

${ }^{\text {II }}$ Curso de Engenharia de Alimentos, DCA, UFLA, Lavras, MG, Brasil.

IIIDCA, UFLA, 37200-000, Lavras, MG, Brasil. E-mail: emramos@dca.ufla.br. *Autor para correspondência.

IvEscola de Nutrição, Universidade Federal de Ouro Preto (UFOP), Ouro Preto, Minas Gerais, Brasil. 
produto final, dependendo do grau de secagem e do teor de lipídeos introduzido inicialmente. Assim, a reformulação desses produtos, seja com redução de lipídeos ou incorporação de substancias com características funcionais, vem sendo amplamente estudadas e indicam resultados promissores (GARCÍA et al., 2002; BACKES, 2008; FERNÁNDEZ-LÓPEZ et al., 2008; KOUTSOPOULOS et al., 2008). GARCÍA et al. (2002), EIM et al. (2008), FERNÁNDEZ-LÓPEZ et al. (2008) relatam a viabilidade da incorporação de fibras na formulação de salames, contribuindo para a redução do teor de lipídeos e do conteúdo calórico, além de ser enriquecido com fibra alimentar.

Muitos subprodutos agroindustriais podem ser incorporados em produtos cárneos para melhorar seus benefícios à saúde, sendo que as fibras de frutas têm apresentados efeitos mais desejáveis na qualidade do produto do que as fibras de cereais (GARCÍA et al., 2002). Nesse sentido, a transformação industrial da uva gera grandes quantidades de subprodutos com elevado teor de fibra alimentar e ricos em compostos bioativos com propriedades antioxidantes (CATANEO et al., 2008).Isso, associado ao seu baixo custo de obtenção, torna seu aproveitamento viável para uso como ingrediente alimentício, conferindo um enriquecimento nutricional e atendendo a objetivos tecnológicos, como a inibição da oxidação lipídica em produtos cárneos.

Nesse contexto, o presente trabalho teve como objetivo verificar o efeito da incorporação de fibras provenientes de subprodutos da elaboração de vinho tinto nas características tecnológicas de salames tipo Milano.

\section{MATERIAL E MÉTODOS}

Os subprodutos provenientes da elaboração de vinho tinto de uvas Vitis vinifera da cultivar 'Syrah', obtida da safra de inverno, foram disponibilizadas pela Empresa de Pesquisa Agropecuária de Minas Gerais (EPAMIG), localizada na cidade de Caldas, MG. A obtenção da farinha foi realizada por meio de secagem em estufa com circulação forçada $\left(60^{\circ} \mathrm{C} / 30 \mathrm{~h}\right)$ e tamização, conforme descrito por NATIVIDADE (2010), sendo avaliadas quanto ao conteúdo de fibra alimentar (solúvel e insolúvel), pela metodologia oficial da AOAC 991.43 (AOAC, 2002), e quanto à atividade antioxidante (\% inibição da oxidação), pelo método da oxidação do $\beta$-caroteno/ácido linoleico descrito por DUARTE-ALMEIDA et al. (2006). Para a atividade antioxidante, foi utilizado como controle o antioxidante sintético Trolox $\left(200 \mathrm{mgL}^{-1}\right)$, conforme sugerido por RUFINO et al. (2006).

A elaboração dos salames seguiu a seguinte formulação básica: carne suína $(69,5 \%)$; toucinho (23\%); sal (2,3\%); sacarose $(0,25 \%)$; glicose $(0,25 \%)$; leite em pó integral $(2,7 \%)$; glucona-delta-lactona $(0,35 \%)$; nitrito de sódio $(0,015 \%)$; acelerador de cura Fixamax-C (0,3\%; New Max Industrial $\left.{ }^{\circledR}\right)$; condimento para salame $\left(0,6 \%\right.$; New Max Industrial $\left.{ }^{\circledR}\right)$; e vinho tinto seco $(1,0 \%)$.

Visando a aumentar o conteúdo de fibra alimentar do produto, e a verificar a contribuição da farinha na prevenção da oxidação dos salames, foram preparados quatro tratamentos a partir da formulação básica: controle, sem adição de farinha e com $23 \%$ de toucinho; $1 \%$ de farinha, com $22 \%$ de toucinho; $2 \%$ de farinha, com $21 \%$ de toucinho; e $3 \%$ de farinha, com $20 \%$ de toucinho.

As carnes foram moídas em discos de $14 \mathrm{~mm}$, os ingredientes misturados e a massa embutida em tripa artificial de celulose (calibre $45 \mathrm{~mm}$ ), sendo armazenados em estufa tipo BOD e mantidos à temperatura média de $25^{\circ} \mathrm{C}$ (umidade relativa média de $90 \%$ ) nos dois primeiros dias e a $15^{\circ} \mathrm{C}$ (umidade relativa média de $83 \%$ ) nos 26 dias restantes , quando os produtos foram considerados prontos.

Para acompanhamento das etapas de fermentação e secagem dos salames, amostragens foram conduzidas nos tempos $0,1,3,5,7,14,21 \mathrm{e}$ 28 dias, sendo realizadas as seguintes análises: perda de peso; $\mathrm{pH}$ e acidez titulável, segundo MATOS et al. (2007); atividade de água, em aparelho Aqualab CX2 (Decagon Devices Inc.); teor de nitrito residual, pelo método oficial no. 973.31 da AOAC (2002); índice de peróxidos (IP), pelo método de PCA-FOX (GAY \& GEBICKI, 2002); e substâncias reativas ao ácido tiobarbitúrico (índice TBARS), segundo RAHARJO et al. (1992). As concentrações de hidroperóxido e de malonaldeído (MDA), para os testes de IP e TBARS, respectivamente, foram determinadas a partir da curva analítica elaborada com hidroperóxidocumeno (CHP) e 1,1,3,3-tetraetoxipropano (TEP). Os produtos acabados foram, ainda, avaliados quanto à composição química (umidade, proteínas, lipídeos e cinzas) de acordo com os métodos oficiais da AOAC (2002).

$\mathrm{O}$ experimento foi realizado em esquema fatorial 4 (formulações) x 8 (tempos), sendo os tratamentos dispostos em delineamento inteiramente casualizado com três repetições, avaliados por meio da análise de variância (ANOVA) e a comparação entre médias realizada pelo teste Tukey, considerando um nível de 5\% de significância. 


\section{RESULTADOS E DISCUSSÃO}

As farinhas obtidas do subproduto da elaboração de vinho continham alto teor de fibra alimentar $(40,26 \%)$, com predominância de fibra insolúvel $(34,40 \%)$, e alta atividade antioxidante $(86,57 \pm 2,12 \%$ de inibição), determinada pelo o método $\beta$-caroteno/ácido linoleico. NATIVIDADE (2010) também verificou alto teor de fibra total $(51,06 \%)$ e insolúvel $(45,28 \%)$, bem como alto poder antioxidante $(91,77 \%$ de inibição), em farinhas provenientes de subprodutos da elaboração de suco de uva (cultivares 'Isabel Precoce' e 'Bordô').

Em relação ao processo de elaboração dos salames, não houve interação significativa $(\mathrm{P}>0,05)$ entre as fontes de variação avaliadas para o $\mathrm{pH}$ e a acidez titulável, entretanto houve $(\mathrm{P}<0,05)$ efeito isolado para os diferentes tratamentos de salame e tempos de processamento. Pôde-se observar uma diminuição nos valores de $\mathrm{pH}$ e, consequentemente, um aumento no conteúdo de ácido lático, à medida que aumentou a concentração de fibras na formulação (Figura 1), diferenciando do controle $(\mathrm{P}<0,05)$. Isso se deve, provavelmente, aos ácidos pré-formados e açúcares fermentáveis presentes na farinha, favorecendo a maior produção de ácido pelas bactérias homofermentativas durante a fermentação. O declínio do $\mathrm{pH}$ durante os primeiros dias de fermentação é fundamental para garantir a inibição de microrganismos indesejáveis, conversão e estabilização da cor e formação de compostos relacionados às características de sabor e aroma (TERRA, 1998; MATOS et al., 2007).

Nos produtos elaborados, o $\mathrm{pH}$ final médio $(4,81 \pm 0,14)$ não foi diferente entre os tratamentos
$(\mathrm{P}>0,05)$, mas os produtos adicionados de farinha apresentaram $(\mathrm{P}<0,05)$ maiores valores de acidez $(2,43 \pm 0,15 \%$ ác. lático $)$ do que o controle $(1,89 \pm 0,16 \%$ ác. lático). A legislação brasileira não define o $\mathrm{pH}$ como parâmetro de qualidade para salames, porém valores próximos do ponto isoelétrico das proteínas é o considerado ideal, uma vez que irá contribuir para perda de umidade e atividade de água do produto.

Da mesma forma que observado para o $\mathrm{pH}$ e acidez, não houve interação significativa $(\mathrm{P}>0,05)$ entre as fontes de variação avaliadas para a perda de peso (PP), embora, para a atividade de água (Aw), a interação tenha sido significativa $(\mathrm{P}<0,05)$. Para $\mathrm{PP}$, houve efeito isolado $(\mathrm{P}<0,05)$ dos níveis de adição da farinha (tratamentos) e do tempo de maturação. Os efeitos da adição de farinha na PP e Aw dos produtos durante a maturação são ilustrados na figura 2 .

Maiores adições de farinha induziram maiores perdas de peso nos produtos acabados (após 28 dias), com o controle apresentando valores médios menores $(32,13 \pm 3,33 \%)$ do que os produtos adicionados de 1 e $2 \%$ de farinha $(35,76 \pm 1,43 \%)$ e com $3 \%$ de farinha $(39,00 \pm 0,56 \%)$. Esta maior perda de peso foi induzida, possivelmente, pelos baixos valores e rápida queda de $\mathrm{pH}$ nos produtos adicionados de farinha. Da mesma forma, a queda na Aw dos produtos foi maior naqueles adicionados de farinha, sendo que, no produto acabado, os salames controle apresentaram Aw de 0,885 $\pm 0,019$, comparado aos $0,850 \pm 0,010$ das amostras adicionadas de 1 e $2 \%$ de farinha e de $0,803 \pm 0,011$ dos produtos adicionados de $3 \%$ de farinha.

Reduções nos valores de Aw também foram observados por EIM et al. (2008), com adição de fibras de cenoura em produtos fermentados (sobrassada). Entretanto FERNÁNDEZ-LÓPEZ et al. (2008) não

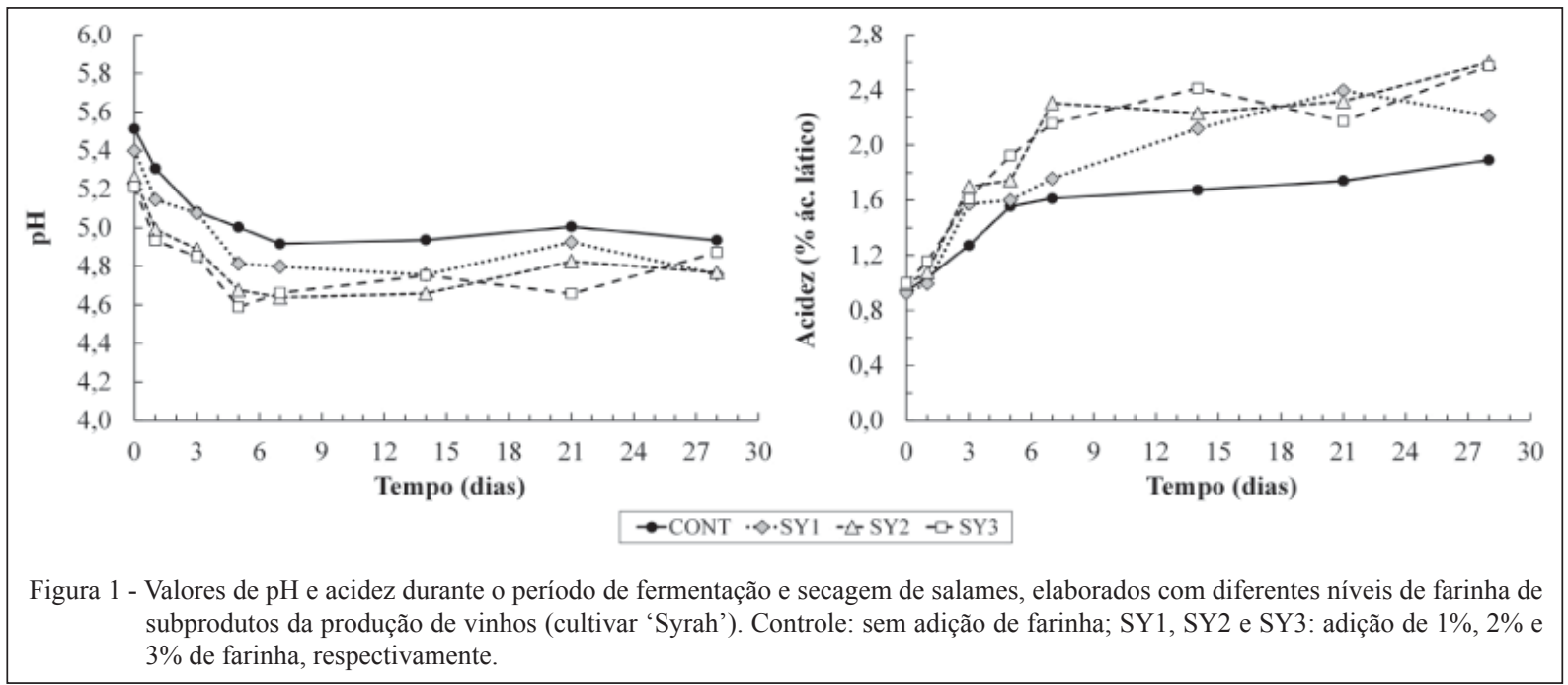

Ciência Rural, v.44, n.7, jul, 2014. 




Figura 2 - Valores de perda de peso e atividade de água durante o período de fermentação e secagem de salames, elaborados com diferentes níveis de farinha de subprodutos da produção de vinhos (cultivar 'Syrah'). Controle: sem adição de farinha; SY1, SY2 e SY3: adição de $1 \%, 2 \%$ e $3 \%$ de farinha, respectivamente.

observaram efeito da adição de fibra de laranja na Aw de salames espanhóis (salchichón), da mesma forma que GARCÍA et al. (2002) não observaram efeitos quando da adição de fibras de cereais (trigo e aveia) e frutas (pêssego, maçã e laranja).

Importante salientar que, independentemente dos efeitos da adição de farinha, todos os produtos atenderam o preconizado pela Instrução Normativa n.22 do Ministério da Agricultura, Pecuária e do Abastecimento (BRASIL, 2000), que estabelece o valor de Aw máximo de 0,900 para o salame tipo Milano. Os valores de Aw encontrados associados ao $\mathrm{pH}$ tornam o produto estável, podendo ser conservados sem refrigeração.

Não foi observado $(\mathrm{P}>0,05)$ efeito da interação entre os tratamentos e os tempos de maturação para os valores residuais de nitrito $\left(\mathrm{NO}_{2} \mathrm{R}\right)$, sendo verificado apenas o efeito $(\mathrm{P}<0,05)$ isolado com a maturação. Já no primeiro dia de fermentação, a concentração de nitrito residual de todos os tratamentos sofreu uma queda brusca, atingindo valores médios de 4,84 $\pm 3,17 \mathrm{ppm}$, permanecendo praticamente constante durante todo processamento


resultado contradiz os relatos de outros autores, que verificaram maiores reduções nos níveis de nitrito residual de produtos cárneos fermentados, à medida que aumentava a quantidade de fibras da laranja (FERNÁNDEZ-LÓPEZ et al., 2008) e limão albedo (ALESON-CARBONELL et al., 2003) adicionada. Por outro lado, é provável que a rápida queda do $\mathrm{pH}$ nos primeiros dias de maturação tenha contribuído para o aumento na velocidade das reações de redução de nitrito (HONIKEL, 2008), fato que pode ter colaborado para não diferença estatística entre os tratamentos e o controle. Isto é reforçado pela alta variação observada na concentração média de nitrito residual $(14,80 \pm 13,48 \mathrm{ppm})$ na massa cárnea logo após embutimento, ocorrida devido a diferenças nos valores médios para os tratamentos. Embora não tenha havido diferença estatística, possivelmente devido à elevada variação, as amostras controle apresentaram valores residuais mais altos $(30,66 \pm 22,66 \mathrm{ppm})$ do que as amostras adicionadas de farinha $(9,41 \pm 7,71 \mathrm{ppm})$.

A evolução das reações de oxidação lipídica nos produtos elaborados foi avaliada medindo-se o teor de hidroperóxidos (índice de peróxidos; IP) e, indiretamente, o teor de aldeídos pela determinação de substâncias reativas ao ácido tiobarbitúrico (TBARS). Para o índice de TBARS, houve efeito $(\mathrm{P}<0,05)$ da interação entre os tratamentos e tempo de maturação, enquanto que, para os valores de IP, foi observado apenas efeito significativo $(\mathrm{P}<0,05)$ com o decorrer do tempo de maturação (Figura 3).

$\mathrm{O}$ teste de IP pelo método PCA-FOX parece não ter sido eficiente para detectar os efeitos relativos à oxidação observados pelo teste de TBARS. É provável que este efeito possa ser devido à presença de nitrito residual no produto. Segundo GAY \& GEBICKI (2002), o método FOX é sensível, sendo baseado na habilidade dos hidroperóxidos em oxidar íons $\mathrm{Fe}^{+2}$ em meio ácido, seguida da formação de um pigmento azul-roxeado resultante da complexação entre os íons $\mathrm{Fe}^{+3}$ formados e o pigmento alaranjado de xilenol (XO). Dessa forma, compostos que se ligam a íons $\mathrm{Fe}^{+3}$, ou que o reduzam a forma ferrosa, como é o caso do nitrito, podem interferir na análise de FOX por competir com o indicador XO. 



$\mathrm{CONT} \cdot \diamond \cdot \mathrm{SY} 1 \quad-\Delta \mathrm{SY} 2 \rightarrow \mathrm{SY} 3$

Figura 3 - Índices de peróxido e TBARS durante o período de fermentação e secagem de salames, elaborados com diferentes níveis de farinha de subprodutos da produção de vinhos (cultivar 'Syrah'). Controle: sem adição de farinha; SY1, SY2 e SY3: adição de $1 \%, 2 \%$ e $3 \%$ de farinha, respectivamente.

A adição de fibras nos salames reduziu significativamente $(\mathrm{P}<0,05)$ a evolução da oxidação lipídica avaliada pelo índice de TBARS, especialmente a partir do décimo quarto dia de maturação (Figura 3). Segundo SAURA-CALIXTO (1998), as fibras encontradas nas uvas são estruturalmente diferentes, podendo ser classificadas como fibras antioxidantes, por estarem associada aos polifenóis, presentes tanto na fração solúvel quanto na fração insolúvel das fibras. Ao final do processamento, após 28 dias, os valores de TBARS no tratamento controle sofreram uma ligeira queda, o que pode ser devido à degradação do malonaldeído (MDA) ao reagir com uma série de compostos, ou mesmo formar dienos e trienos de MDA, diminuindo sua quantidade disponível para reagir com o ácido tiobarbitúrico (GRAU et al., 2001).

Os dados relativos à composição centesimal dos salames acabados estão representados na tabela 1 . A umidade, lipídeos e proteína dos salames contendo fibras não diferiram significativamente do tratamento controle $(\mathrm{P}>0,05)$. Apesar de não significativo, o aumento no teor de lipídeos dos tratamentos contendo farinha provavelmente ocorreu devido à maior perda de água nesses produtos, evidenciada pela redução nos valores médios da umidade. Todos os tratamentos atenderam aos valores estabelecidos pela legislação brasileira para salame tipo Milano (BRASIL, 2000), para a umidade (máximo de $35 \%$ ) e proteína (mínimo de $23 \%$ ).

Considerando a adição de farinha de subprodutos da produção de vinho nos níveis de 1,2 e $3 \%$, os produtos acabados (após a secagem) continham 1,56, 3,11 e 4,92\% de farinha, respectivamente. Dessa forma, baseado no teor de fibra alimentar $(40,26 \%)$ presente na farinha, estima-se que os salames elaborados com 1,2 e $3 \%$ de farinha continham, quando acabados, 0,63, 1,25 e $1,98 \%$ de fibra alimentar total, respectivamente. Embora esses valores possam ser considerados baixos, deve-se atentar ao fato de que, especialmente para um produto isento de fibras, representam um aumento significativo nas propriedades nutricionais dos produtos.

Tabela 1 - Média ( \pm desvio padrão) da composição centesimal de salames elaborados com diferentes níveis de farinha de subprodutos da produção de vinhos (cultivar 'Syrah'), após 28 dias de processamento.

\begin{tabular}{lllll}
\hline Amostras & Umidade & Lipídeo & Proteína & Cinza \\
\hline Controle & $34,56 \pm 6,17$ & $32,10 \pm 5,37$ & $24,25 \pm 0,74$ & $3,30^{\mathrm{b}} \pm 0,24$ \\
SY1 & $33,15 \pm 1,62$ & $30,38 \pm 1,94$ & $27,07 \pm 0,93$ & $3,83^{\mathrm{a}, \mathrm{b}} \pm 0,40$ \\
SY2 & $31,80 \pm 2,02$ & $34,10 \pm 2,24$ & $25,44 \pm 1,72$ & $3,61^{\mathrm{a}, \mathrm{b}} \pm 0,21$ \\
SY3 & $28,60 \pm 0,55$ & $34,37 \pm 1,18$ & $24,92 \pm 0,31$ & $4,26^{\mathrm{a}} \pm 0,29$ \\
\hline
\end{tabular}

Médias com letras diferentes em uma mesma linha são estatisticamente diferentes pelo teste de Tukey (P<0,05). Controle: sem adição de farinha; SY1, SY2 e SY3: adição de $1 \%, 2 \%$ e $3 \%$ de farinha, respectivamente. 


\section{CONCLUSÃO}

Salames podem ser enriquecidos nutricionalmente pela adição de fibras obtidas de subprodutos da produção de vinho tinto, além de contribuir para o processo de fermentação e maturação do produto e reduzir os efeitos da oxidação lipídica, característicos em produtos cárneos fermentados.

\section{AGRADECIMENTOS}

Os autores agradecem à Fundação de Amparo à Pesquisa do estado de Minas Gerais (FAPEMIG), pelo apoio financeiro à execução do projeto, à A Coordenação de Aperfeiçoamento de Pessoal de Nível Superior (CAPES), pela bolsa de mestradopara o primeiro autor, e ao O Conselho Nacional de Desenvolvimento Científico e Tecnológico (CNPq), pela bolsa PIBIC do segundo autor.

\section{REFERÊNCIAS}

ALESON-CARBONELL, L. et al. Utilization of lemon albedo in dry-cured sausages. Journal of Food Science, v.68, p.18261830, 2003. Disponível em: <http://onlinelibrary.wiley.com/ doi/10.1111/j.1365-2621.2003.tb12337.x/pdf>. Acesso em: 10 fev. 2014. doi: 10.1111/j.1365-2621.2003.tb12337.x.

AOAC (ASSOCIATION OF OFFICIAL ANALYTICAL CHEMISTS). Official methods of analysis. 17.ed. Gaithersburg, 2002. $2000 \mathrm{p}$.

BRASIL. Ministério da Agricultura, Pecuária e Abastecimento (MAPA). Secretaria de Defesa Agropecuária (SDA). Instrução Normativa n.22, 31 julho de 2000.Aprova os Regulamentos Técnicos de Identidade e Qualidade de Jerked Beef, de Presunto tipo Parma, de Presunto Cru, de Salame, de Salaminho, de Salame tipo Alemão, de Salame tipo Calabres, de Salame tipo Friolano, de Salame tipo Napolitano, de Salame tipo Hamburgues, de Salame tipo Italiano, de Salame tipo Milano, de Linguiça Colonial e Pepperoni. Diário Oficial da União, Brasília, seção 1, p.15-28, 2000.

CATANEO, C.B. et al. Atividade antioxidante e conteúdo fenólico do resíduo agroindustrial da produção de vinho. Semina: Ciências Agrárias, v.29, n.1, p.93-102, 2008. Disponível em: $<$ http://www.uel.br/revistas/uel/index.php/semagrarias/article/ view/2856/2424>. Acesso em: 03 fev. 2013.

DUARTE-ALMEIDA, J.M. et al. Avaliação da atividade antioxidante utilizando sistema $\beta$-caroteno/ $\alpha$ cido linoléico e método de seqüestro de radicais DPPH•. Ciência e Tecnologia de Alimentos, v.26, n.2, p.446-452, 2006. Disponível em: <http:// www.scielo.br/pdf/cta/v26n2/30196.pdf $>$. Acesso em: 03 fev. 2013. doi: 10.1590/S0101-20612006000200031.

EIM, V.S. et al. Effects of addition of carrot dietary fibre on the ripening process of a dry fermented sausage (sobrassada). Meat Science, v.80, p.173-182, 2008. Disponível em: <http://www. sciencedirect.com/science/article/pii/S0309174007003890\#>. Acesso em: 10 fev. 2014. doi: 10.1016/j.meatsci.2007.11.017.

FERNÁNDEZ-LÓPEZ, J. et al. Physico-chemical and microbiological profiles of "salchichón" (Spanish dry-fermented sausage) enriched with orange fiber. Meat Science, v.80, p.410417, 2008. Disponível em: <http://www.sciencedirect.com/
science/article/pii/S0309174008000065>. Acesso em: 10 fev. 2014. doi: 10.1016/j.meatsci.2008.01.010.

GARCÍA, M.L. et al. Utilization of cereal and fruit fibres in low fat dry fermented sausages. Meat Science, v.60, p.227-236, 2002. Disponível em: < http://www.sciencedirect.com/science/article/pii/ S0309174001001255>. Acesso em: 10 fev. 2014. doi: 10.1016/ S0309-1740(01)00125-5.

GAY, C.A.; GEBICKI, J.M. Perchloric acid enhances sensitivity and reproducibility of the ferric-xylenol orange peroxide assay. Analytical Biochemistry, v.304, p.42-46, 2002. Disponível em: <http://www. sciencedirect.com/science/article/pii/S0003269701955666>. Acesso em: 10 fev. 2014. doi: 10.1006/abio.2001.5566.

GRAU, A. et al. Oxidative stability of dark chidken meat through frozem storage: influence of dietary fat and $\alpha$-tocopherol and ascorbic acid supplementation. Poultry science, v.80, n.11, p.1630-1642, 2001. Disponível em: <http://www.poultryscience. org/ps/paperpdfs/01/p01B1630.pdf>. Acesso em: 08 mar. 2013.

HONIKEL, K.O. The use and control of nitrate and nitrite for the processing of meat products. Meat Science, v.78, p.68-76, 2008. Disponível em: <http://www.sciencedirect.com/science/article/pii/ S0309174007001994>. Acesso em: 10 fev. 2014. doi: 10.1016/j. meatsci.2007.05.030.

MATOS, R.A. et al. Efeito do tipo de fermentação na qualidade final de embutidos fermentados cozidos elaborados a base de carne ovina. Boletim Ceppa, v.25, p.225-234, 2007. Disponível em: $<$ http://ojs.c3sl.ufpr.br/ojs2/index.php/alimentos/article/view/ 10610/7092>. Acesso em: 08/02/2013.

NATIVIDADE, M.M.P. Desenvolvimento, caracterização e aplicação tecnológica de farinhas elaboradas com resíduos da produção de suco de uva. 2010. 202f. Dissertação (Mestrado em Ciência dos Alimentos) - Universidade Federal de Lavras, Lavras, MG.

RAHARJO, S. et al. Improved speed, specificity, and limit of determination of an aqueous acid extraction thiobarbituric acid-C18 method for measuring lipid peroxidation in beef. Journal of Agricultural and Food Chemistry, v.40 n.11, p.21822185, 1992. doi: 10.1021/jf00023a02.

RODRÍGUEZ, R. et al. Dietary fibre from vegetable products as source of functional ingredients. Trends in Food Science and Technology, v.17, p.3-15, 2006. Disponível em: <http://www. sciencedirect.com/science/article/pii/S092422440500244X >. Acesso em: 10 fev. 2014. doi: 10.1016/j.tifs.2005.10.002.

RUFINO, M.S.M. et al. Metodologia científica: determinação da atividade antioxidante total em frutas no sistema $\beta$-caroteno/ acido linoléico. Fortaleza: EMBRAPA, 2006. 4p. (Comunicado técnico, 126).

SAURA-CALIXTO, F. Antioxidant dietary fiber product: a new concept and a potential food ingredient. Journal of Agricultural and Food Chemistry, v.46, n.10, p.4303-4306, 1998. Disponível em: < http://pubs.acs.org/doi/pdf/10.1021/jf9803841>. Acesso em: 10 fev. 2014. doi: 10.1021/jf9803841.

TERRA, N.N. Apontamentos de tecnologia de carnes. São Leopoldo: Unisinos, 1998. 216p.

VERMA, A.K.; BANERJEE, R. Dietary fibre as functional ingredient in meat products: a novel approach for healthy living - a review. Journal of Food Science \& Technology, v.47, n.3, p.247257, 2010. Disponível em: <http://link.springer.com/ article/10.1007\%2Fs13197-010-0039-8\#page-1>. Acesso em: 10 fev. 2014. doi: 10.1007/s13197-010-0039-8. 